\title{
The Study of Accuracy Characteristics of Information Extraction System Under Conditions of Change of State of the Working Signals Propagation Path and A-Priori Uncertainty About the Informative Parameters of Objects on the Sighting Surface
}

\author{
Oleh Vorobiov ${ }^{1}$, NataliiaYeromina $^{2}$, Sergey Petrov ${ }^{3}$, Oleksandr Ivashcuk ${ }^{4}$, Vitaly Ivansky ${ }^{5}$, Mykola \\ Pavlunko $^{6}$, SerhiiNevhad ${ }^{7}$ \\ ${ }^{1}$ Department of Logistics, Logistics Institute, the National Defense University of Ukraine \\ Povitroflotsky, Kyiv, Ukraine, oleg33377@ukr.net \\ ${ }^{2}$ Department of Electronic Computers, Kharkiv National University of Radio Electronics, Ukraine, Kharkiv, \\ nataliia.yeromina@nure.ua \\ ${ }^{3}$ Department of Physics, Electrical Engineering and Power Engineering, Ukrainian Engineering Pedagogics \\ Academy, Ukraine, psv_topol@ukr.net \\ ${ }^{4}$ National Defense University of Ukraine named after Ivan Cherniakhovskyi, Ukraine, sashik-ivashuk84@ukr.net \\ ${ }^{5}$ National Academy of Land Forces named after hetman Petro Sagaidachny, Lviv, Ukraine, Vet658@ukr.net \\ ${ }^{6}$ Department of operation and combat support of Troops (Forces) Support and Information Technologies, National \\ Defense University of Ukraine named Ivan Cherniakhovskyi, n.pavlunko@gmail.com \\ ${ }^{7}$ Language Testing and Research Centre of the Foreign Languages Education and Research Centre of the National \\ Defence University of Ukraine, serhii_nevhad @ nuou.org.ua
}

\begin{abstract}
The solution of the characteristics of an information extraction system under conditions of stochastic change of the state of the working signals propagation path and apriori uncertainty about the informative parameters of objects on the sighting surface.

The main ratios and sequences of the required evaluations are given. Numerical estimates of the accuracy characteristics of the information extraction systems in case of navigation of aircrafts have been obtained.

It has been established that deterioration of the signal on the propagation path significantly affects the accuracy of navigation of aircraft at high altitudes, and at the last frame at an altitude of about $500 \mathrm{~m}$, deterioration can be neglected. It is shown that at the noise-to-signal ratio $\mathrm{q} \approx 3$ , the navigation accuracy is no fewer than $10 \mathrm{~m}$.
\end{abstract}

Key words: information extraction systems, interference attack, engineering activities, sighting surface, accuracy characteristics.

\section{INTRODUCTION}

Process of operation of earth surface monitoring systems, remote objects detection systems, navigation systems of aircrafts, which belong to the class of remote information extraction systems, depends on a multitude of stochastic factors, which can significantly reduce their effectiveness. Accidental or intentional interference with the class of systems under consideration may be caused by interference from different generation systems, electronic warfare system (EWS). Primarily, they affect the receiver pathways as well as the environment in which the operating signals of the considered systems are disseminated. Moreover, the various engineering activities carried out at a location used by the information extraction systems will result in changes in the sighting surface, which will also reduce their efficiency. One of the most important quality indicators for the systems under consideration is the accuracy indicator, which will also be stochastic taking into account the stochastic conditions of operation. Therefore, the question of the exact characteristics of remote information extraction systems remains a pressing one.

\subsection{Problem analysis}

The study of the accuracy characteristics of the information extraction systems is described in works [1-6], in which it was assumed that a priori information on the informative parameters of the measured sighting surface is not available. The influence of the signal propagation medium on the accuracy of the systems was not considered. The work [7] proposes a solution to the problem of estimating the potential accuracy of an aircraft navigation system in terms of the theory of signal parameter estimation.Inpointoffact,

theinteractionoftheoperationalsignalofthenavigationsystem withnoise, thestatisticalcharacteristicsofwhichareknown, isconsidered.It was necessary to find an optimal estimate of the unknown signal parameter $\varepsilon$ with unknown interference parameters $\mathrm{T}_{\mathrm{o}}$, and $\mathrm{T}_{\phi}$. In such a case, the criterion of maximum likelihood was used as the criterion of optimality. However, the influence of the signals propagation path condition was not taken into consideration. 
At the same time, it is possible to have another research objective, which is to assess the potential accuracy of the navigation system by assuming that the parameters of the background are known, in particular, its temperature $T_{\phi}$, while the temperature of the object $\mathrm{T}_{\mathrm{o}}$ is unknown and is to be assessed, as well as the contrast $\Delta \mathrm{T}=\mathrm{T}_{\mathrm{o}}-\mathrm{T}_{\phi}$ and likelihood functions.

Since the background of the sighting surface is usually the same in engineering activities, it is interesting to study the accuracy characteristics of the information extraction system in the conditions above stated in the research objective.

The object of the article is to study the accuracy characteristics of the information extraction system, taking into account the influence of the signal propagation medium, as well as the change of the informative parameter of the reference object.

\section{MAIN MATERIAL}

2.1 The study of the accuracy characteristics of the information extraction system with respect to the influence of the signals propagation path

2.1.1 Results of experimental studies on the influence of the propagation medium on the image of the sighting surface formed by the information extraction system

Images of the sighting surface are obtained with the Dji Mavic Pro quadcopter, which is equipped with a gyrostabilised $4 \mathrm{~K}$ camera. The appearance of the quadcopterDji Mavic Pro and its control panel are shown in Figure1.
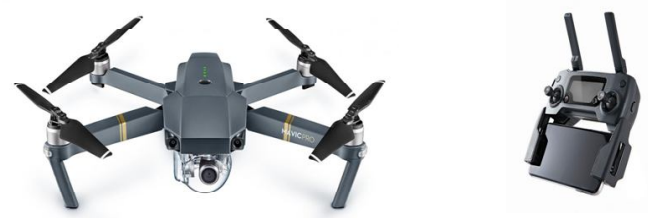

Figure 1:Exterior of the quadcopter and its control panel

In order to visually assess the quality of the images, depending on the influence of the propagation medium of the working signals, images from different altitudes were obtained in a clean atmosphere and low fog. Images of the sighting surface are shown in Figures2, 3.

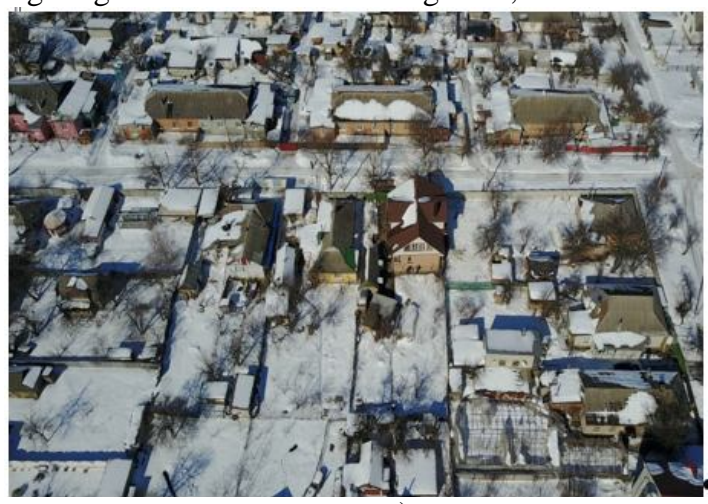

a)

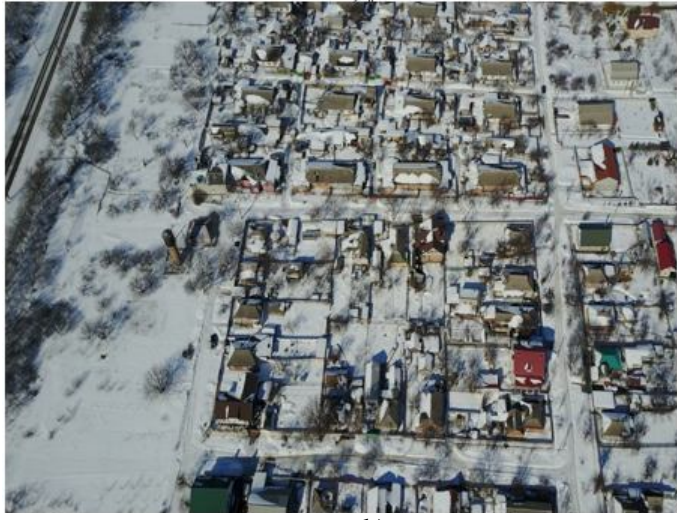

b)

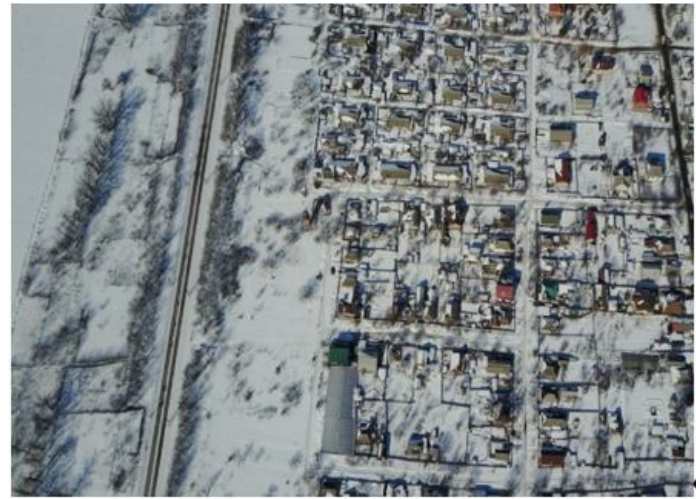

c)

Figure 2:Images of the sighting surface in a clean atmosphere from altitudes:

a) $100 \mathrm{~m}$; b) $250 \mathrm{~m}$; ) $500 \mathrm{~m}$

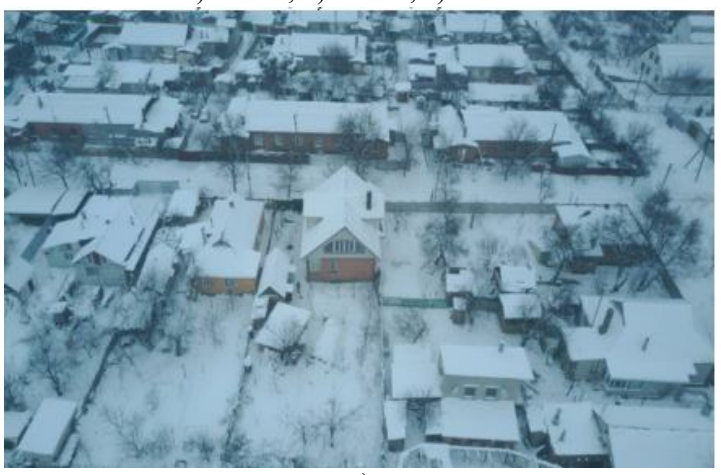

a)

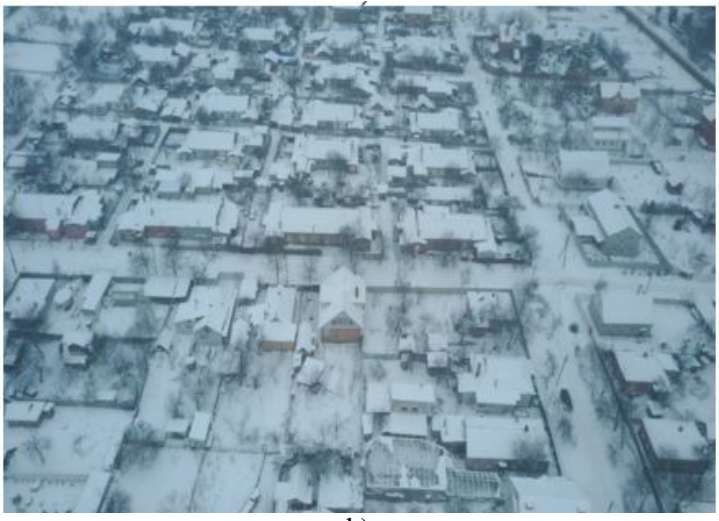

b) 


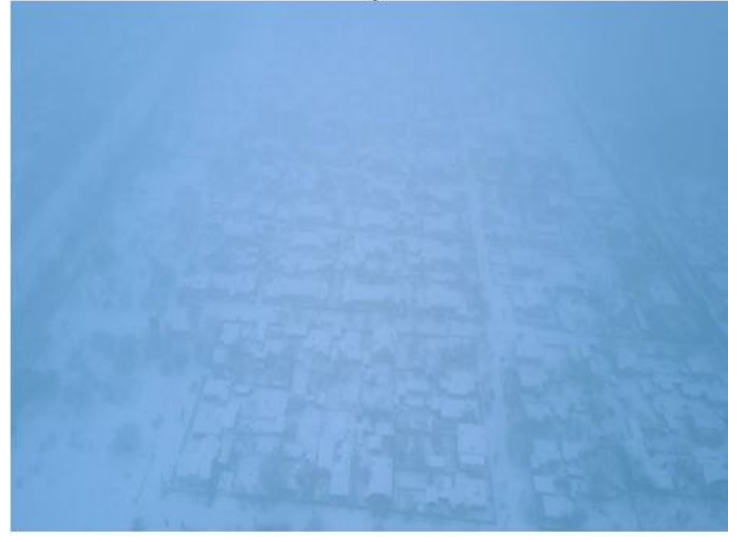

c)

Figure 3:Images of sighting surface in mild fog from altitudes: a) $50 \mathrm{~m}$; b) $100 \mathrm{~m}$; c) $200 \mathrm{~m}$

The visual analysis of the above images points to the need to take into consideration the impact of the signals propagation path on the accuracy characteristics of the information extraction system.

2.1.2 Study of the accuracy characteristics of the information extraction system in the event of a change in the state of the propagation path of the working signals

In accordance with the results of the work [7], accuracy can be estimated by defining and calculating correspondences $\sigma_{\varepsilon}(\varepsilon), \sigma_{\mathrm{T}_{\mathrm{o}}}(\varepsilon), \sigma_{\mathrm{T}_{\phi}}(\varepsilon)$ for ratios for mean-square deviations of the parameters being evaluated:

$$
\begin{aligned}
& \sigma_{\varepsilon}=\frac{|\sin \langle\mathbf{f}, \mathbf{e}\rangle|}{\Delta \mathrm{T}\left\|\mathbf{f}_{\mathcal{E}}\right\| \mathrm{F}\left(\mathbf{f}, \mathbf{e}, \mathbf{f}_{\mathcal{E}}\right)} ; \\
& \sigma_{\mathrm{T}_{\mathrm{o}}}=\frac{\left|\sin \left\langle\mathbf{f}_{\mathcal{E}}, \mathbf{e}\right\rangle\right|}{\|\mathbf{f}\| \mathrm{F}\left(\mathbf{f}, \mathbf{e}, \mathbf{f}_{\mathcal{E}}\right)} ; \\
& \sigma_{\mathrm{T} \phi}=\frac{\left|\sin \left\langle\mathbf{f}_{\mathcal{E}}, \mathbf{e}\right\rangle\right|}{\|\mathbf{e}\| \mathrm{F}\left(\mathbf{f}, \mathbf{e}, \mathbf{f}_{\mathcal{E}}\right)},
\end{aligned}
$$

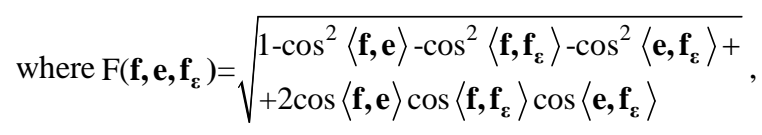

$\cos \langle\mathbf{f}, \mathbf{e}\rangle$ isthecosineanglebetweenvectorsf ande .

Figure 4 illustrates the graph of accuracy $\sigma_{\varepsilon}$ against the signal-noise ratio $\mathrm{q}$ at a fixed parameter value $\varepsilon=\mathrm{x}_{41}\left(\mathrm{t}_{0}\right)-\mathrm{x}_{0} \approx 172,22 \mathrm{~m}$, which coincides with the position of the axis of the antenna directivity diagram (ADD) of the forth beam (lower curve) and the above mentioned set of other parameters.

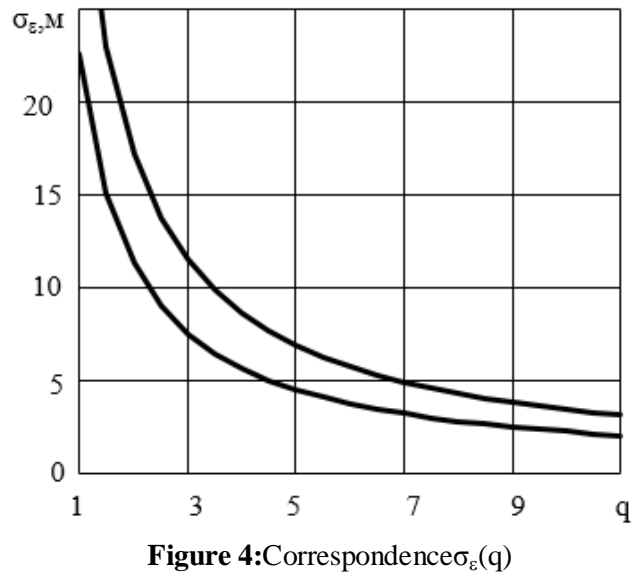

The upper curve corresponds to the value

$$
\varepsilon=\left[\mathrm{x}_{41}\left(\mathrm{t}_{0}\right)+\mathrm{x}_{51}\left(\mathrm{t}_{0}\right)\right] / 2-\mathrm{x}_{0},
$$

i.e. the position of the temperature difference coincides with the midpoint of the junction of the ADD axes of the fourth and fifth beams of the antenna with the surface. Furthermore, the channel noise dispersion $\sigma^{2}$ and background temperature $\mathrm{T}_{\phi}$ were assumed to be constant, and the temperature $\mathrm{T}_{\mathrm{o}}$ has been changed for noise-to-signal ratio. For the navigation of aircrafts, it is desirable that the ratioq $>3$. It follows from the graph that for images in which the reference object occupies a significant part of the sensor's field of view, there may be a significant improvement in accuracy compared to there solution of the element of the antenna system on the ground. Thus, for example, at the mean distance between the axes of the adjacent $\mathrm{ADD} \approx 20 \mathrm{~m}$ for signal-noise ratioq=5...9 the navigation accuracy is $\sigma_{\varepsilon}=(4,5 \ldots 2,5) \mathrm{m}$.

Figure 4's graph does not account for the attenuation of the signal on the propagation path, which causes the signal-tonoise ratio to deteriorate. To illustrate this effect on Figure 5 , a correspondence set is shown of $\sigma_{\varepsilon}$ against altitude $z_{0}$, frame exposure for different values of linear attenuation on the propagation path, which was taken into account by a known factor [1]:

$$
\mathrm{L}=\exp \left[-\alpha \mathrm{z}_{0} \sec \varphi\right] \text {, }
$$

where $\alpha$ is the long attenuation of signal, $\mathrm{dB} / \mathrm{km}$.

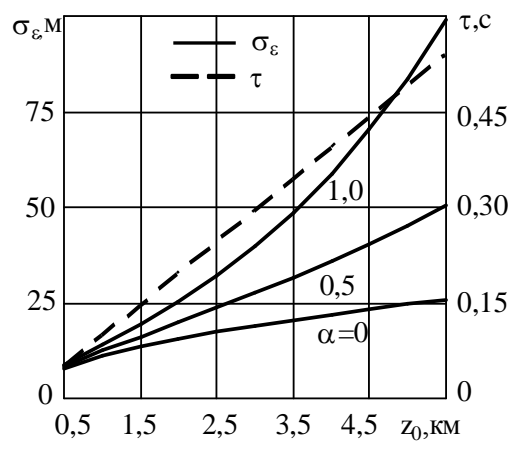

Figure 5: Correspondence $\sigma_{\varepsilon}\left(\mathrm{z}_{0}\right), \tau\left(\mathrm{z}_{0}\right)$ 
Oleh Vorobiov et al., International Journal of Emerging Trends in Engineering Research, 8(9), September 2020,5740 - 5745

The altitude values in formula (3) shall be expressed in kilometres. Calculations are performed according to the first expression of the correspondence (1), where the contrast is $\Delta \mathrm{T}$ should be replaced by $\vartheta \mathrm{T}=\mathrm{L} \Delta \mathrm{T}$.

For each altitude according to the formula:

$$
\tau=\frac{\Delta_{\mathrm{x}_{1}} \cos \left(\varphi-\beta_{1}\right)}{3 \mathrm{~V} \sin \beta_{1}}
$$

a valid constant time value $\tau$ is calculated.

Correspondence $\tau\left(\mathrm{z}_{0}\right)$ is also represented in the Figure 6 by bar line.

The contrast-forming temperatures were chosen to be equal to $\mathrm{T}_{\phi}=270 \mathrm{~K}, \mathrm{~T}_{\mathrm{o}}=260 \mathrm{~K}$.

Value of the temperature difference $\varepsilon$ co-ordinate at each altitude value $z_{0}$ was chosen from the condition (2).Long attenuation $\alpha=1 \mathrm{~dB} / \mathrm{km}$ corresponds to the attenuation of radio waves with a wavelength of $8.6 \mathrm{~mm}$ at a rain intensity of $4 \mathrm{~mm} / \mathrm{h} \mathrm{[1]}$.

Analysis of the graphs in Figure 5 shows that signal attenuation on the propagation path significantly affects the accuracy of navigation of aircraft at high altitudes, and at the last shot at high altitude $\approx 500 \mathrm{~m}$, attenuation may be neglected.

2.2 Study of the accuracy characteristics of an information extraction system under conditions of a priori uncertainty about the informative parameters of objects on the sighting surface

In the case of a priori uncertainty about the informative parameters of objects on the sighting surface the expression for the accuracy characteristics of the evaluation are written as follows:

$$
\begin{aligned}
& \sigma_{\varepsilon}=\left[\left\|\mathbf{f}_{\varepsilon}\right\|^{2}-\left(\mathbf{f}_{\varepsilon}, \mathbf{f} /\|\mathbf{f}\|\right)^{2}\right]^{-1 / 2} / \Delta T, \\
& \sigma_{\Delta \mathrm{T}}=\left[\|\mathbf{f}\|^{2}-\left(\mathbf{f}, \mathbf{f}_{\varepsilon} /\left\|\mathbf{f}_{\varepsilon}\right\|\right)^{2}\right]^{-1 / 2} .
\end{aligned}
$$

If the parameter $\Delta \mathrm{T}$ isalso be considered as known, then

$$
\sigma_{\varepsilon}=1 /\left(\Delta \mathrm{T}\left\|\mathbf{f}_{\varepsilon}\right\|\right)
$$

According to the general theory, the accuracy of the joint assessment of parametereshould drop as the number of parameters assessed increases. In fact, the accuracy of navigation calculated by the formulas (1), (5), (7) for the set of parameters used in the construction of Figure 5, and for the parametere, determined by the condition (2), was (atq=3) $\sigma_{\varepsilon}=11,477 \mathrm{~m}, \sigma_{\varepsilon}=10,659 \mathrm{~m}, \sigma_{\varepsilon}=9,926 \mathrm{~m}$ respectively.
Let us consider the case when a range $\left\{a_{1} \leq x \leq b_{1}\right\} \times\{y \in \mathbf{R}\}$ with a known width $\Delta \mathrm{l}=\mathrm{b}_{1}-\mathrm{a}_{1}$ is used as a reference object. Neglecting the phenomenon of blur, i.e. assuming the channel to be inertialless, we represent the output signal of the channel as:

$$
\mathrm{f}_{\mathrm{i}}(\varepsilon)=\frac{1}{\sigma_{\mathrm{i}}}\left[\Phi\left(\frac{\varepsilon+\Delta \mathrm{l}-\zeta_{\mathrm{i}}}{\delta_{\mathrm{i}}}\right)-\Phi\left(\frac{\varepsilon-\zeta_{\mathrm{i}}}{\delta_{\mathrm{i}}}\right)\right]
$$

$$
\frac{\partial \mathrm{f}_{\mathrm{i}}(\varepsilon)}{\partial \varepsilon}=\frac{1}{\sqrt{2 \pi} \sigma_{\mathrm{i}} \delta_{\mathrm{i}}}\left\{\begin{array}{l}
\exp \left[-\frac{\left(\varepsilon+\Delta \mathrm{l}-\zeta_{\mathrm{i}}\right)^{2}}{2 \delta_{\mathrm{i}}^{2}}\right]- \\
-\exp \left[-\frac{\left(\varepsilon-\zeta_{\mathrm{i}}\right)^{2}}{2 \delta_{\mathrm{i}}^{2}}\right]
\end{array}\right\},
$$

where $\varepsilon=\mathrm{a}_{1}-\mathrm{x}_{0}, \mathrm{i} \in \overline{1, \mathrm{~N}_{1}}$.

Figure 6 shows the relation graph between the accuracy of navigation and the position of the border $a_{1}$ of the range of $\Delta \mathrm{l}=20 \mathrm{~m}$, constructed according to formula (1).Comparing to the case of a half-space for a given range width, a slightly higher accuracy and the appearance on the curve of another additional minimum corresponding to the coordinate of the axis of the ADD of the first beam can be noticed.

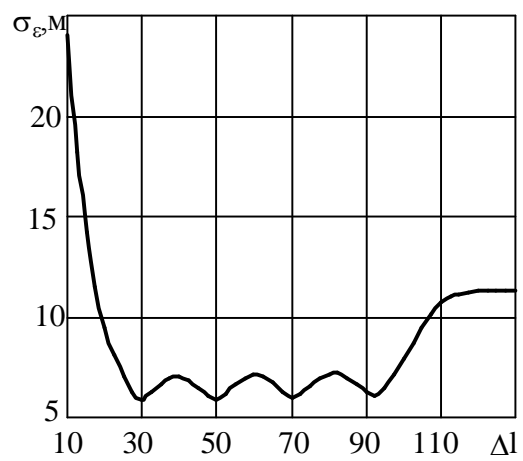

Figure 6: Correspondence $\sigma_{\varepsilon}(\varepsilon)$

Figure 7 illustrates the correspondence of navigational accuracy to range width.

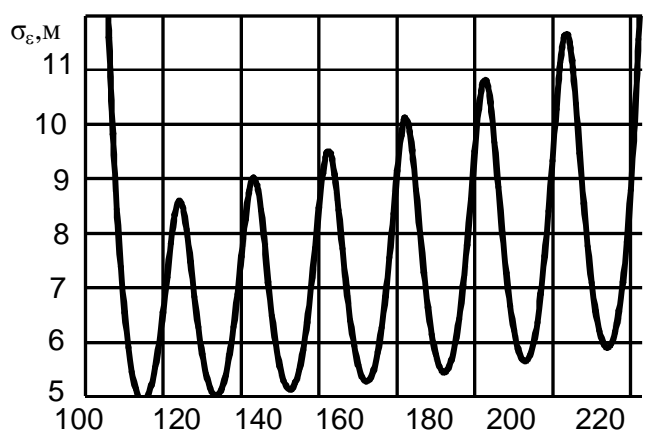

Figure 7:Correspondence $\sigma_{\varepsilon}(\Delta \mathrm{l})$ 
Oleh Vorobiov et al., International Journal of Emerging Trends in Engineering Research, 8(9), September 2020,5740 - 5745

When the width of the range changes, the curves like the one shown in Figure 5 become slightly offset; for each width value $\Delta l$ the position $\varepsilon$ of the third maxima of the curve in Figure 5 was calculated and function values $\mathrm{f}_{\mathrm{i}}(\varepsilon), \frac{\partial \mathrm{f}_{\mathrm{i}}(\varepsilon)}{\partial \varepsilon}$ were calculated for the found position using formulas (8), (9) and then using an expression (1) the value of the accuracy of navigation was determined. The mean of the half-power semi-axle of the ellipse was calculated as $\Delta_{\mathrm{X}} \approx 10 \mathrm{~m}$, and an average distance between the axes of adjacent axes $\mathrm{ADD}$ is equal tothe value $2 \Delta_{\mathrm{X}}$. The position of the third curve maxima in the Figure 5 for the different values $\varepsilon$ was determined to lie in the range of [160...168] $\mathrm{m}$.

Analysis of the graph leads to the following conclusions:

1) In area $\varepsilon<2 \Delta_{x}$ as the $\varepsilon$ decreases, the accuracy of the navigation drops sharply;

2) Inarea $\varepsilon>110$ mthe second boundary of the range $b_{1}=\varepsilon+\Delta l+x_{0}$ does not enter the field of view of the system and the range is perceived by the sensor as a half-space, therefore the accuracy of the navigation corresponds to the received in work [1] for the halfspace and is equal (for the third maximum) to $\sigma_{\varepsilon} \approx 9,5$ $\mathrm{m}$;

3) In the intermediate area, the accuracy of navigation is the best, since both boundaries of the range enter the viewing area and it is maximal for $\varepsilon \approx(2 \mathrm{k}+1) \Delta_{\mathrm{x}}, \mathrm{k} \in \overline{1,4}$, i.e. the width of the range is an odd number of half-intervals of the resolution of the antenna system.

\section{CONCLUSION}

Experimental studies of the image quality dependency of the retrieval system show the need to take into account signal attenuation caused by the influence of the propagation path.

Theoreticalestimatesoftheaccuracycharacteristicsofinformat ionextractionsystemsincaseofnavigationofaircraftshavesho wnthat deterioration of the signal on the propagation path significantly affects the accuracy of navigation of aircraft at high altitudes, and at the last frame at an altitude of about $500 \mathrm{~m}$, deterioration can be neglected. The solution of the problem is suggested in order to determine the accuracy characteristics of an information extraction system under conditions of a-priori uncertainty about the informative parameters of objects on the sighting surface.

It is shown that at the noise-to-signal ratio $\mathrm{q} \approx 3$ the navigation accuracy is no fewer than $10 \mathrm{~m}$.

\section{REFERENCES}

1. V. Antyufeev. Matrix radiometric correlationextreme navigation systems for aircraft: monograph Ukraine, Kharkov: KhNU V.N. Karazin, 372 p.

2. A. Sotnikov, V. Tarshyn, N. Yeromina, S. Petrov, N. Antonenko. A method for localizing a referenceobject in a current image with several bright objects, EasternEuropean Journal of Enterprise Technologies. 2017. Vol.
№ 9 (87).pp. $\quad 68-74 . \quad$ DOI: $10.15587 / 1729-$ 4061.2017.101920.

3. N.Yeromina, S. Petrov, A. Tantsiura, M. Iasechko, V. Larin. Formation of reference images and decisionfunction in radiometric correlation-extremal navigation systems. Eastern-European Journal ofEnterprise Technologies. - 2018. Vol.4, No.9 (94). pp. 27-35. DOI: 10.15587/1729-4061.2018.139723.

4. O. Sotnikov, V. Kartashov, O.Tymochko, O.Sergiyenko, V.Tyrsa, P.Mercorelli, W. Flores-Fuentes. Methodsforensuringtheaccuracyof radiometricandopto electronicnavigationsystemsofflyingrobotsin

a developed

5. Sotnikov O. etal. (2020)

MethodsforEnsuringtheAccuracyofRadiometricandOptoe lectronicNavigationSystemsofFlyingRobotsin a

DevelopedInfrastructure. In: Sergiyenko O., Flores-

Fuentes W., Mercorelli P. (eds)

MachineVisionandNavigation. Springer, Cham.

https://doi.org/10.1007/978-3-030-22587-2_16

6. N.Yeromina, S. Petrov, A. Tarasenko, N. Antonenko, O. Semenenko, O. Pavlovskyi, M. Sliusarenko, V. TelehinThe Use of Strong and Ordinal Scales during the Synthesis of Reference Images for Vehicle Correlation-Extreme, IJATCSE, 9 (2), 2020, pp. 23432349, doi: 10.30534/ijatcse/2020/218922020

7. A. Tantsiura, D. Kolomiets, I. Tabakova, I. Hannoshyna, N. Serdiuk, O. Yelieazarov, T. Voichenko. EvaluationofthePotentialAccuracyofCorrelationExtre meNavigationSystemsof Low-Altitude MobileRobots, IJATCSE, 8 (5), 2019, pp. 2161-2166, doi:

10.30534/ijatcse/2019/47852019

8. S. Trehubenko, L. Berkman, N. Yeromina, S. Petrov, Y. Bryzhatyi, H. Kovalov, V.Dachkovskyi, L.MikhailovaThe Operation of Detection Systems in Conditions of Contrast Decrease of Ground Objects, JETER, 8 (1), 2020, pp. 208-212, doi: 10.30534/ijeter/2020/28812020

9. N. Yeromina, S. Petrov, N. Antonenko, I. Vlasov, V. Kostrytsia, V. KorshenkoThe Synthesis of the Optimal Reference Image Using Nominal and Hyperordinal Scales, JETER, 8 (5), 2020, pp. 2080-2084, doi: 10.30534/ijeter/2020/98852020

10. O. Liashko, V. Klindukhova, N.Yeromina, T.Karadobrii, O.Bairamova, A. DoroshevaThe Criterion and Evaluation of Effectiveness of Image Comparison in Correlation-Extreme Navigation Systems of Mobile Robots, JETER, 8 (6), 2020, pp. 2841-2847, doi: 10.30534/ijeter/2020/ 97862020

11. N.Yeromina, V. Samoilenko, D. Chukanivskyi, O. Zadkova, O. Brodova, O. Levchenko The Method of Iterative Formation of Selective Reference Images, JETER, 8 (7), 2020, pp. 3753-3759, doi: 10.30534/ijeter/2020/138872020

12. N.Yeromina, S. Petrov, Y. Samsonov, S. Pisarevskiy, S.Kaplun, I. VlasenkoThe Simulation and Performance Evaluation of Adaptive Algorithm of Image Comparison in Correlation-Extreme Navigation Systems, JETER, 8 (8), 2020, pp. 4146-4151, doi: 10.30534/ijatcse/2020/19882020

13.O.Tymochko , A.Ttystan , V.Ushan , N.Yeromina , O.Dmitriiev, V.Mazharov, I.Padalka , I.Hannoshyna , I. Masik, A. ZazirnyiThe Synthesis of the Reference Image 
and Algorithms for Vehicle Navigation Systems,

JETER,8(3), 2020, pp. 853-858. doi:

10.30534/ijeter/2020/40832020.

14. O.Tymochko, A.Trystan, A.Berezhnyi, O.Sotnikov, O.Matiushchenko, I.Kryzhanivskyi Vehicles while Monitoring Behaviorof DynamicObjectsin a ForestSteppe Area,JETER,8(7), 2020, pp. 32083215.doi.org/10.30534/ijeter/2020/54872020

15. O. Vorobiov, V. Savchenko, A. Sotnikov, V.Tarshin, and T. Kurtseitov. Development of radioisotopic-plasma technology for the protection ofradio electronic means from powerful electromagnetic radiation. EasternEuropean Journalof Enterprise Technologies. 2017 Vol. № 5(85), pp. 16-22.doi:10.15587/1729-4061.2017.91642. 